\title{
Using Polaris and the Big Dipper to approximate local standard time
}

\author{
Edwin H. Kaufman Jr. ${ }^{1^{*}}$, Corey R. Page ${ }^{2}$, Casey R. Watson ${ }^{3}$ \\ ${ }^{1}$ Department of Mathematics, Central Michigan University, Mount Pleasant, USA; \\ *Corresponding Author: edkaufman@mac.com \\ ${ }^{2}$ Department of Mechanics, Materials, and Aerospace Engineering, Illinois Institute of Technology, Chicago, USA \\ ${ }^{3}$ Department of Physics and Astronomy, Millikin University, Decatur, USA
}

Received 30 September 2012; revised 30 October 2012; accepted 14 November 2012

\begin{abstract}
In this paper we introduce methods for approximating local standard time in the Northern Hemisphere using Polaris and the Big Dipper as well as alternative reference stars, and describe in detail how to construct a device we call a dipperclock to facilitate this process. An alternative method which does not require a dipperclock is also discussed. Ways of constructing dipperclocks which glow in the dark are presented. The accuracy of dipperclocks is examined, both theoretically and through field testing. A java program is provided for creating dipperclocks customized to a particular year-long time period and place to get improved accuracy. Basic astronomical definitions and justifications of the results are provided. We also discuss the use of dipperclocks to find longitude and latitude.
\end{abstract}

Keywords: Polaris; Big Dipper; Time; Longitude; Latitude

\section{INTRODUCTION}

The North Star Polaris and the Big Dipper can be thought of as a giant clock in the sky, with the imaginary hour hand of the clock extending from Polaris through the pointer stars in the bowl of the Big Dipper (see Figure 1). One needs to imagine a 24-hour clockface centered at Polaris which runs counterclockwise, is perpendicular to your line of sight to Polaris, and has midnight at the top and noon at the bottom. The time indicated in the picture appears to be about 10:30 a.m., before adjusting for the date, which we will do in Appendix 8.

\footnotetext{
${ }^{1}$ Patent pending.

${ }^{2}$ Stars by Stellarium (developed by Fabien Chéreau, Matthew Gates, Nigel Kerr, Diego Marcos, Bogdan Marinov, Timothy Reaves, Alexander Wolf, Guillaume Chéreau, and Barry Gerdes). Hour hand by photoshop.
}

Since the earth rotates clockwise on its axis when viewed from below the solar system, the Big Dipper, which provides the hour hand for our clock, appears to rotate counterclockwise about Polaris. References [1-7] rely on the use of imaginary 24 or 12-hour clockfaces that the reader must envision in the sky to estimate the sidereal time. To get local standard time, a mathematical calculation involving the date is needed in $[2-4,6,7]$. We describe a similar "equinox estimation method" in Appendix 8. Reference [1] uses a mechanical translator to do the calculation. References [8-12] discuss mechanical devices for telling time by the Big Dipper and other stars. We note that our dipperclock ${ }^{1}$ differs from the devices we have found in the literature in various ways, including the following: 1) The dipperclock has no parts which move relative to one another; thus it is simpler and easier to make than the other devices we have found in the literature. As shown in Figure 2, the dipperclock consists of a single disk with a hole in the center; 2) The dipperclock can be made in glow-in-the-dark versions, which are easier to read in dark areas. In Figure 2, the numbers

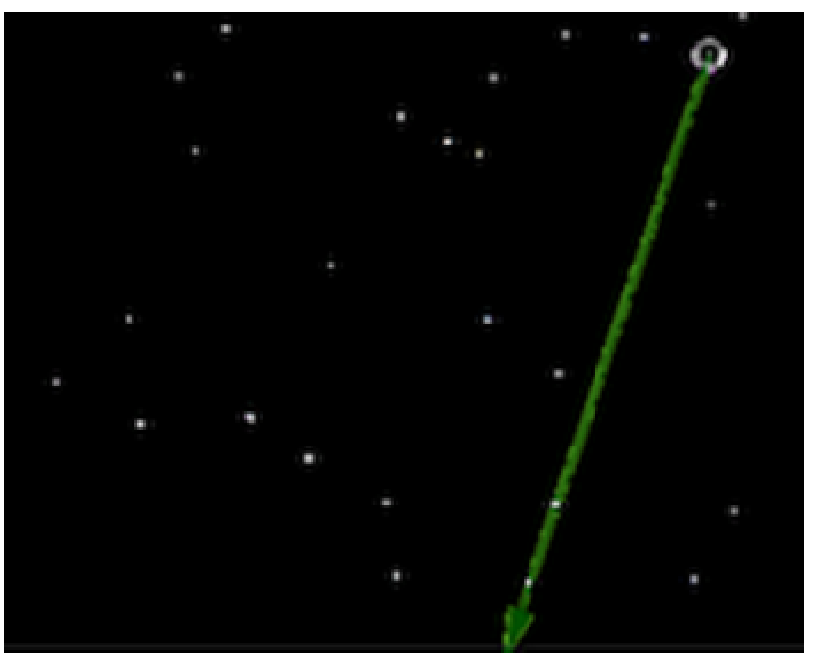

Figure 1. The celestial clock². 


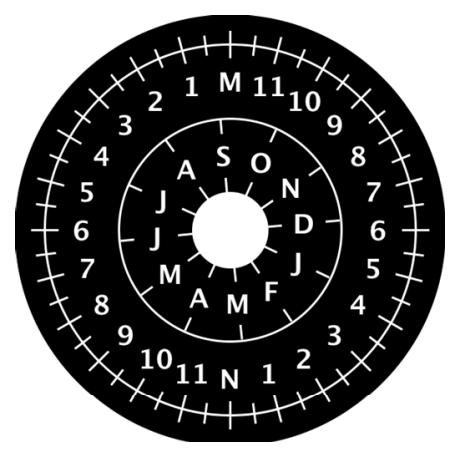

Figure 2. The generic dipperclock.

and letters, the concentric circles, and the hash marks can be made to glow in the dark; 3) Custom dipperclocks can be generated using the free java program which we are supplying; 4) This paper supplies proofs for the claims we make about the dipperclock. Some of the proofs are quite intricate because the clookface, which is the outer ring in Figure 2, rotates when the date changes.

In Section 3 we discuss a correction based on the user's longitude that can improve the accuracy of the dipperclock. In Section 4 we show how a dipperclock can be used as a longitude finder if the Universal Time (see Appendix 1) and date are known, and can be used to find the latitude in the Northern Hemisphere as well. In Section 5 we discuss glow-in-the-dark dipperclocks.

We relegate many of the details of our work to the following 12 Appendices:

Appendix 1: A list of basic astronomical definitions.

Appendix 2: Justification of the geographic correction.

Appendix 3: Justification of the method of using a dipperclock to find longitude.

Appendix 4: Using different stars as the tip of the hour hand.

Appendix 5: Discussion of a free java program to generate dipperclocks.

Appendix 6: Construction of glow-in-the-dark dipperclocks.

Appendix 7: Field testing.

Appendix 8: Finding the time without a dipperclock (the equinox estimation method).

Appendix 9: Discussion of the mathematical foundations required for Appendices 10 - 12.

Appendix 10: Discussion of how the various numbers and hash marks are placed around the dipperclock.

Appendix 11: Accuracy of the dipperclock. (Bottom line: Under typical assumptions, theoretical error $<13$ minutes for a generic dipperclock assuming a geographic correction is done; theoretical error $<5$ minutes for a custom dipperclock.)

Appendix 12: Accuracy of the equinox estimation method. (Bottom line: Under typical assumptions, theoretical error $<16$ minutes assuming a geographic correc- tion is done.)

\section{THE DIPPERCLOCK}

Figure 2 is a diagram of our generic dipperclock (By a generic dipperclock we mean a dipperclock which assumes the previous autumnal equinox to be midnight in Universal Time on September 22, ignores leap years, and does not have a built-in geographic correction; such a dipperclock can be used at any time.) In the diagram, the letters and numbers in the outer ring represent local standard time, with the $\mathrm{M}$ representing midnight, the numbers 1 through 11 running counterclockwise from $M$ representing a.m. times, the $\mathrm{N}$ representing noon, and the numbers 1 through 11 running counterclockwise from $\mathrm{N}$ representing p.m. times. Each letter in the inner ring represents the first day of a month, with the months running clockwise; for example, the $\mathrm{F}$ in the inner ring represents February 1, the $M$ in the inner ring adjacent to the F represents March 1, etc. When the dipperclock is used (as described in more detail below) the user rotates the dipperclock so that the current date is at the bottom of the inner ring, positions the dipperclock so that Polaris is centered in the center of the central hole of the dipperclock, and reads the local standard time indicated in the outer ring by the "pointer stars" in the bowl of the Big Dipper.

This diagram can also be obtained by going to the URL in [13]. To make a working version, either copy Figure 2 onto a stiff piece of paper or print the diagram from [13]. Then, cut away the part inside the inner ring and the part outside the outer ring, leaving a doughnutshaped object consisting of two rings containing letters, numbers and hash marks. The numbers in the outer ring represent local standard time, with $\mathrm{M}$ denoting midnight and $\mathrm{N}$ denoting noon. The letters in the inner ring represent the first days of months at midnight. For example, F represents February 1 at midnight.

Recommended viewing procedure:

1) If you are using a glow-in-the-dark dipperclock (Section 5), expose it to light before using it.

2) Locate the Big Dipper (see Figure 1 or Figure 3).

3) Locate the North Star Polaris by drawing an imaginary line through the "pointer stars" in the bowl of the Big Dipper farthest from the handle.

4) Hold your dipperclock with today's date in the inner ring directly below the center.

5) Center Polaris in the hole.

6) Move your dipperclock back and forth along the line from your eye to Polaris, keeping it perpendicular to this line, until the pointer stars are close to the outer ring.

Then read the standard time at the point on the outer ring closest to the pointer stars.

7) If you are on daylight savings time, add one hour.

Example 1 


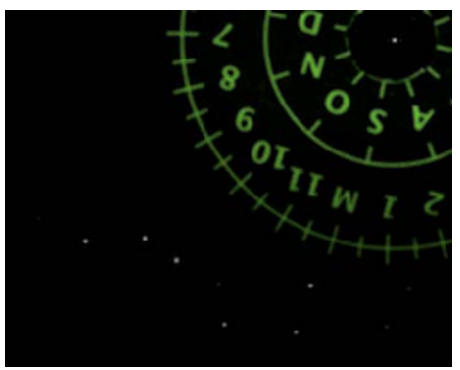

Figure 3. Illustration for example $1^{3}$.

Suppose that on August 17 we view Polaris and the Big Dipper using a $203 \mathrm{~mm}(8 \mathrm{inch})$ glow-in-the-dark dipperclock (Section 5) and what we see is as shown in Figure 3 above. Note that the dipperclock has been rotated so that August 17 in the inner ring is at the bottom of the dipperclock. The pointer stars in the bowl of the Big Dipper give a reading of about 11:50 p.m. local standard time, which is about 12:50 a.m. local daylight time.

\section{GEOGRAPHIC CORRECTION}

Your location within your time zone could cause an error of a half hour or more in the time read from your dipperclock. To fix this problem, let

$\mathrm{GC}=$ the geographic correction (in minutes);

LST $=$ your local standard time (in hours);

$\mathrm{UT}=$ the Universal Time (in hours) (see Appendix 1);

$\mathrm{L}=$ your longitude (in degrees).

Then

$$
\mathrm{GC}=4 \times(15 \times(\mathrm{LST}-\mathrm{UT})-\mathrm{L})
$$

This formula is justified in Appendix 2.

Note that LST - UT is constant throughout your time zone. In any time zone, we call the line of longitude of $15 \times($ LST - UT $)$ degrees the centerline of the time zone, even though it might not be in the center. West longitude is taken to be negative, and east longitude is taken to be positive. We see from Eq.1 that the geographic correction is zero if you are located on the centerline of your time zone.

As an example, we will consider the geographic correction for Chicago, Illinois, which is located in the U.S. Central Time Zone at west longitude $87.63^{\circ}$ (see [14]). LST - UT $=-6$, which follows from the fact that Central Standard Time is 6 hours behind UT, or one can get UT from [15] (or http://www.usno.navy.mil) and compute LST - UT directly. From Eq.1, we have

${ }^{3}$ Stars by Stellarium (developed by Fabien Chéreau, Matthew Gates, Nigel Kerr, Diego Marcos, Bogdan Marinov, Timothy Reaves, Alexander Wolf, Guillaume Chéreau, and Barry Gerdes). Combining of stars and DSLR photo of letter-size glowing dipperclock, and brightness enhancement of dipperclock photo, by photoshop.

$$
\begin{aligned}
\mathrm{GC} & =4 \times(15 \times(-6)-(-87.63)) \\
& =4 \times(-90+87.63) \\
& =-9.48 \text { minutes } \\
& =-9 \min 29 \mathrm{sec}
\end{aligned}
$$

so we must subtract about 9 minutes from our time estimate for Chicago.

\section{USING A DIPPERCLOCK AS A LONGITUDE AND LATITUDE FINDER}

Given the date, time zone, and longitude, we have shown how one can use a dipperclock to find the local standard time (LST). If instead the Universal Time and date are known, one can then use a dipperclock to find the longitude. If we let DT (in hours) = the local standard time estimate found by the dipperclock without a geographic correction, it is shown in Appendix 3 that your longitude in degrees is given by

$$
\mathrm{L}=(\mathrm{DT}-\mathrm{UT}) \times 15
$$

In the Northern Hemisphere, it is also possible to use Polaris to find your north latitude (which equals the angle of elevation of Polaris above the northern horizon [2]), and therefore completely specify your location. To use your dipperclock to approximately find this angle, hold the dipperclock vertically with the central hole beside your left eye and the $\mathrm{M}$ in the outer ring lined up with the northernmost point on your horizon, then read the nearest position on the outer ring to Polaris and multiply this value by 15 .

If the errors in the time indicated by a dipperclock, which we discuss at length in Appendix 11, amount to $\mathrm{m}$ minutes (i.e. $\mathrm{m} / 60$ hours), then by Eq.2, they will cause an error of $\mathrm{m} / 4$ degrees in the estimate of the longitude.

Although we have not covered celestial navigation in any depth in this paper, the interested reader can find much more information about it in $[16,17]$.

\section{Example 2}

Question: Suppose a dipperclock constructed with no geographic correction says that the local standard time is 9:27 p.m., and at the time this reading is taken the Universal Time is 05:37 (i.e. 5:37 a.m.). Suppose also that when the dipperclock is held vertically with the central hole beside your left eye and the $\mathrm{M}$ in the outer ring lined up with the northernmost point on your horizon, Polaris is near the 2.5 in the outer ring. Where are you?

Answer: By Eq.2,

$$
\begin{aligned}
\mathrm{L} & =(-8: 10) \times 15 \\
& =\left(-8 \frac{1}{6}\right) \times 15 \\
& =-122.5^{\circ}
\end{aligned}
$$




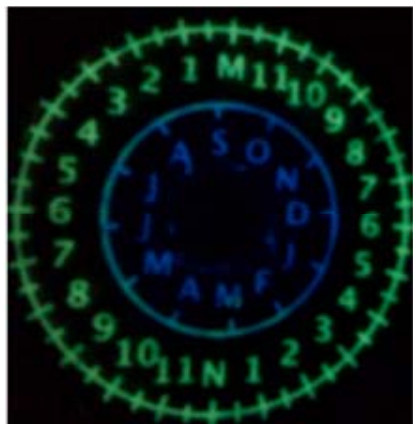

Figure 4. $76 \mathrm{~mm}$ (3 inch) dipperclock $^{4}$.

that is, the west longitude is $122.5^{\circ}$. Your latitude is about $2.5 \times 15=37.5^{\circ}$. Checking a map or [14] shows that you are in or near San Francisco, California (which has west longitude $122.4^{\circ}$ and latitude $37.8^{\circ}$ ).

\section{GLOW-IN-THE-DARK DIPPERCLOCKS}

In a very dark area that is otherwise ideal for night sky viewing, it may be difficult to see the numbers on the dipperclock. We have used phosphorescent paper or paint to deal with this problem. The construction of glow-inthe-dark dipperclocks is discussed in Appendix 6. A phosphorescent paint dipperclock is shown in Figure 4.

\section{REFERENCES}

[1] Collins, E.B. (1926) Useful stargazing. Scientific American, 134, 58-61.

[2] Kaufman Jr., E.H. (2011) How to find celestial objects by starhopping.

http://dl.dropbox.com/u/32602966/starhopping.pdf

[3] Mammana, D. (1990) How to tell time by the big dipper. http://www.dennismammana.com/skyinfo/gazingtips/dipp er time.htm

[4] Miles, K.A. and Peters, C.F. II (1997) What the big dipper can tell you. http://starryskies.com/articles/dln/4-97/dipper.time.html

[5] Pasachoff, J.M. (2000) A field guide to the stars and planets. 4th Edition, Houghton Mifflin, New York.

[6] Layton, B. and Huffman, A. (1999) Telling time by the big dipper.

http://www.physics.ucla.edu/ huffman/dtime.html

[7] Wylie, C.C. and Merrymon, W.W. (1926) Time by the big dipper. Popular Astronomy, 34, 229.

http://articles.adsabs.harvard.edu/full/seri/PA.../0034//000 0231.000.html

[8] The Editors of Sky and Telescope (2011) Make a star wheel! Sky and Telescope.

http://www.skyandtelescope.com/letsgo/familyfun/Make a Star Wheel.html

[9] Chai, Q.H., Henry C.S.S., Tan C.Y. and Wong S.L. GEK 1506, heavenly mathematics: Cultural astronomy, group 32 methods of telling time. National University of Singapore, Singapore.

http://www.math.nus.edu.sg/aslaksen/gem-projects/hm/05 06-1-32-Methods to tell time.pdf

[10] Jen, Dr. and Van Dresar, J. (2008) Nocturnal time dial part II: Getting the time using the big dipper. 4Physics. http://www.4physics.com/phy demo/NocturnalTimeDial/ BigDipperTime.html

[11] Schatz, D. and Allen, P. (2011) Make a star clock! Sky and Telescope.

http://www.skyandtelescope.com/letsgo/familyfun/Make a Star Clock.html

[12] Uses of the astrolabe. http://www.astrolabes.org/pages/uses.htm

[13] Kaufman Jr., E.H., Page, C.R. and Watson, C.R. (2012) Dipperclock. http://dl.dropbox.com/u/32602966/dipperclock.TIFF

[14] Latitude and longitude of a point. Google Maps. http://itouchmap.com/latlong.html

[15] US Naval Observatory (2000) Earth's seasons: Equinoxes, perihelion, solstices, and aphelion, 2000-2020.

http://www.usno.navy.mil/USNO/astronomical-applicatio ns/data-services/earth-seasons

[16] Favill, J. (1940) Primer of celestial navigation. Cornell Maritime Press, New York.

[17] Nassau, J. (1948) Practical astronomy. McGraw Hill, New York.

[18] Kaufman Jr., E.H. (2011) A java program for drawing dipperclocks. http://dl.dropbox.com/u/32602966/dipperclock.java

[19] Java download for a PC. (2009) http://www.oracle.com/technetwork/java/javase/downloa ds/index.html

[20] Eck, D.J. (2010) Introduction to programming using java. 5th Edition. http://math.hws.edu/javanotes/index.html

[21] Glow in the dark photo paper. (2011) http://www.amazon.com/Glow-Dark-Photo-Paper-pack/d $\mathrm{p} / \mathrm{B} 003 \mathrm{EM} 7 \mathrm{TP} 0 / \mathrm{ref}=\mathrm{sr} \_1$ 1 ie $=\mathrm{UTF} 8 \& q \mathrm{id}=1308068515$ $\underline{\& s r}=8-1$

[22] Glow in the dark paint. (2011) http://www.glowinc.com

[23] Timekeeping. (2010) http://csep10.phys.utk.edu/astr161/lect/time/timekeeping. $\underline{\mathrm{html}}$
${ }^{4}$ Pictures taken by iPhone app Tripod (Jeff McMorris). Rotation and resizing by MAC programs keynote and preview. 


\section{APPENDIX 1: A LIST OF BASIC ASTRONOMICAL DEFINITIONS}

In this section we give a few definitions from Astronomy along with some discussion of some of them. These definitions can also be found in [2,5] and many other places.

a) In Astronomy, the sphere of the sky surrounding the earth is called the CELESTIAL SPHERE, where the center of the earth is also the center of the celestial sphere. We give distant stars fixed positions on the celestial sphere called declination (D) and right ascension (RA), which are similar to latitude and longitude on an earth map.

b) A GREAT CIRCLE on the celestial sphere is a circle formed by cutting the celestial sphere with a plane that contains the center of the earth. These are the largest possible circles on the sphere.

c) The CELESTIAL EQUATOR is the great circle formed by cutting the celestial sphere with the plane that contains the center of the earth and is perpendicular to earth's rotation axis.

d) The NORTH (SOUTH) CELESTIAL POLE is the point where the northern (southern) portion of the earth's axis meets the celestial sphere. The north celestial pole is close to Polaris; the south celestial pole is close to the Southern Cross.

e) RIGHT ASCENSION: We lay out a distance scale on the celestial equator by dividing it into 24 one-hour intervals by means of points which are numbered 0 to 23 , moving clockwise as viewed from below the solar system. The zero point is located in the constellation Pisces. For any object or point on the celestial sphere other than a celestial pole, there is one and only one great circle that contains the celestial poles and this object or point. The RIGHT ASCENSION of the object or point is defined relative to the closest point where this great circle intersects the celestial equator.

f) UNIVERSAL TIME (UT) is approximately standard time at the Royal Observatory in Greenwich, England, and was formerly known as GREENWICH MEAN TIME (GMT). UT is 5 hours ahead of standard time in the U.S. Eastern time zone (EST). There is also COORDINATED UNIVERSAL TIME (UTC) which is measured by atomic clocks but which is kept within 0.9 seconds of UT by the addition of an occasional "leap second". For the purposes of this paper we will treat UTC and UT as being the same.

g) The ZENITH is the highest point in the sky, directly overhead.

h) One's MERIDIAN is the part of a great circle that starts at the north celestial pole, passes through one's zenith, and ends at the southernmost point on one's horizon.

i) One's SIDEREAL TIME is the right ascension of whatever is on one's meridian at the time.

j) AUTUMNAL EQUINOX: Between summer and fall, we reach a point in our orbit about the sun when the plane which contains the earth's equator hits the center of the sun. As a result, the day and night that occur closest to this point are of roughly equal length. This is called the autumnal equinox. (Equinox $=$ equi $($ equal $)+$ nox (night)). A table of autumnal equinoxes for the years 2000-2020 is given in [15] in UT. From [15] we see for instance that the autumnal equinox for 2010 was 3:09 UT September 23, or September 22 at 10:09 p.m. (EST) or 11:09 p.m. (EDT).

\section{APPENDIX 2: JUSTIFICATION OF THE GEOGRAPHIC CORRECTION}

We wish to justify Eq.1 in Section 3, which is repeated here as

$$
\mathrm{GC}=4 \times(15 \times(\mathrm{LST}-\mathrm{UT})-\mathrm{L})
$$

where

$\mathrm{GC}=$ the geographic correction (in minutes);

LST $=$ your local standard time (in hours);

$\mathrm{UT}=$ the Universal Time (in hours) (see Appendix 1);

$\mathrm{L}=$ your longitude (in degrees).

Using Eq.A1 is equivalent to the pair of statements (A1a) and (A1b) below:

If you are east of the centerline, then for every degree of longitude you are east of the centerline, subtract 4 minutes from your time estimate (A1a); and

If you are west of the centerline, then for every degree of longitude you are west of the centerline, add 4 minutes to your time estimate (A1b).

This equivalence follows from the facts that the absolute value of $15 \times($ LST - UT) - L is just the number of degrees of longitude between your location and your centerline, with $15 \times($ LST - UT) - L being negative if you are east of your centerline and being positive if you are west of your centerline.

Now it remains only to show that statements (A1a) and (A1b) give a valid time correction. Suppose you are one degree of longitude east of your centerline. Instead of thinking of moving one degree east from the centerline, equivalently think of the earth suddenly turning one degree clockwise (as viewed from below) with you on it. This will make the Big Dipper appear to rotate one degree counterclockwise. Since there are 360 degrees in a full circle, this rotation will be $1 / 360$ of a full circle, so regardless of what 24-hour clockface you are using, the hour hand will advance $(1 / 360) \times 24$ hours, which is $(1 / 360) \times(24 \times 60)$ minutes, which is 4 minutes. Since you are still in the same time zone, your local standard time has not changed, so to compensate you will need to subtract 4 minutes from your time estimate. The argument for the case where you are west of the centerline is 
similar.

\section{APPENDIX 3: JUSTIFICATION OF THE METHOD OF USING A DIPPERCLOCK TO FIND LONGITUDE}

In this appendix we wish to verify the equation

$$
\mathrm{L}=(\mathrm{DT}-\mathrm{UT}) \times 15
$$

where

$\mathrm{L}=$ your longitude (in degrees);

DT $=$ the local standard time estimate found by the dipperclock without a geographic correction (in hours);

$\mathrm{UT}=$ the Universal Time (in hours) (see Appendix 1).

Since GC is what we add to DT to get LST, we have

$$
(\mathrm{LST}-\mathrm{DT})(\text { in minutes })=\mathrm{GC}(\text { in minutes })
$$

so from Eq.A1,

$$
\begin{aligned}
& (\mathrm{LST}-\mathrm{DT})(\text { in minutes }) \\
= & 60 \times((\mathrm{LST}-\mathrm{UT})(\text { in hours }))-4 \times \mathrm{L}
\end{aligned}
$$

Converting the left side to hours, we get

$$
\begin{aligned}
& 60 \times((\text { LST }-D T)(\text { in hours })) \\
= & 60 \times(\text { LST }-\mathrm{UT})(\text { in hours })-4 \times \mathrm{L}
\end{aligned}
$$

Dividing by 60 , we get

$$
(\mathrm{LST}-\mathrm{DT})(\text { in hours })=(\mathrm{LST}-\mathrm{UT})(\text { in hours })-\mathrm{L} / 15
$$

Canceling LST, we get

$$
- \text { DT }(\text { in hours })=- \text { UT }(\text { in hours })-\text { L/15 }
$$

Transposing terms, we get

$$
\mathrm{L} / 15=(\mathrm{DT}-\mathrm{UT})(\text { in hours })
$$

Finally, multiplying by 15 , we get

$$
\mathrm{L}=(\mathrm{DT}-\mathrm{UT})(\text { in hours }) \times 15
$$

which is what we wanted to show.

\section{APPENDIX 4: USING DIFFERENT STARS AS THE TIP OF THE HOUR HAND}

Until now we have used the pointer stars in the bowl of the Big Dipper as the tip of the celestial hour hand because the Big Dipper is familiar to most people and is easy to find. Other bright stars that are close to Polaris can also be used as the tip of the celestial hour hand, however. We simply need to utilize their right ascension (RA) rather than the right ascension of the bowl stars of the Big Dipper (for which RA $=11$ hours). The equinox estimation method also works, if we replace the 11 in the adjustment term with the RA of the new star (see Appendix 8).
One reasonable choice for the tip of the hour hand is Kocab (aka $\beta$ UMi). In Figure 1, Kocab is the brightest star left of and a little below Polaris. Kocab was also mentioned in [7], and is the brightest star in the bowl of the Little Dipper. Kocab has the advantage that it is closer to Polaris than the pointer stars in the Big Dipper are, so it may be visible when the pointer stars are blocked by trees, for instance. For Kocab, we have RA = 14 hours 50.7 minutes $=14.845$ hours.

Another reasonable choice would be $\gamma$ Cas, which is the middle star in the $\mathrm{W}$ of Cassiopeia. $\gamma$ Cas has the advantage that it is on the opposite side of Polaris from the Big Dipper, so it will be high in the sky when the Big Dipper may be too low to be visible. For $\gamma$ Cas we have $\mathrm{RA}=56.7$ minutes $=0.945$ hours .

If a different tip star is desired, find its RA and use the java program in Appendix 5 to construct an appropriate dipperclock, or use the equinox estimation method presented in Appendix 8 with RA in place of 11. The RAs of many other good candidates can be found in Table 2 of [5].

It is also possible to use a dipperclock constructed for the Big Dipper with the new tip star; just take a reading with the new tip star and add RA - 11 hours. This procedure will be justified in Appendix 10. For example, if $\mathrm{RA}=17$ hours then you can take a reading with the new tip star and add 6 hours.

\section{APPENDIX 5: DISCUSSION OF A FREE JAVA PROGRAM TO GENERATE DIPPERCLOCKS}

We have written a standalone java program which will generate a dipperclock according to one's specifications and put it in a png file of one's choosing. We are providing the source code for this program free in the text file dipperclock.java, which you can download from [18]. This file was created using the Mac text editor TextWrangler.

To compile and run this program, you will need to have JDK (Java Development Kit) installed. Note that JRE (Java Runtime Environment) is not sufficient. JDK should already be present on Macs running OS 10.5 or higher; for PCs it can be downloaded for free from [19] (the leftmost of the four download icons in [19] should work well).

To launch the program, open a command window. On a Mac, use the Terminal program; on a Windows computer, use the cmd program; and with Linux, use Konsole, gterm, or xterm.

After creating a folder called javawork in your home directory and saving dipperclock.java there, type the following three commands in the command window:

cd javawork;

javac dipperclock.java; 
java dipperclock.

One should not need the command window again except to show error messages, if there are any. For further information, see Sections 2.6.1 and 2.6.2 of [20]. The program should now execute the following series of dialog boxes:

1) Tip Stars: You will be asked whether you want to use the pointer stars in the bowl of the Big Dipper as the tip of the dipperclock's hour hand. If you say no, you will then be asked to give the right ascension of the alternative star(s) you would like to use instead.

2) Year-Pair: You will be asked for the first year of the year-pair. The reason for the term year-pair is that a dipperclock involves parts of two consecutive calendar years, namely from the autumnal equinox of the first year to the autumnal equinox of the second year. If you give any negative number, you will get a generic dipperclock.

3) LST - UT: You will be asked for the difference between your local standard time (LST) and Universal Time (UT). Note that this difference might not be an integer; for example, Newfoundland is $31 / 2$ hours behind UT, so LST - UT $=-3.5$ in Newfoundland. Our methods still work in such cases. If you are doing a generic dipperclock with no geographic correction, then LST - UT will not be needed by the program, so you can select anything.

4) GC: You will be asked whether you want to do a geographic correction (GC), and if so, you will be asked for your longitude (which can be found using [14]).

5) Size: You will be asked to specify the size of your dipperclock.

6) Color: You will be asked to specify the color pattern of your dipperclock. You can choose white on black or black on white, or you can independently choose the color of the background, the three circles, the dates and their hash marks, and the times and their hash marks. In each case your choices are black, white, red, blue, green, and yellow.

7) Confirm: The program will give a summary of your choices, and ask you if you are satisfied; if not, you will be taken through steps $1-7$ again.

8) Storage: The program will ask where you would like to save the .png file containing your dipperclock.

If you choose, you may change the source code for this program. Additionally, you may find some of the subroutines to be useful independently; for example, FindAEUT and Isleap can be used to estimate the autumnal equinox for a given year in Universal Time. See the comments (preceded by // or bracketed by $/ * * /$ ) in dipperclock.java for more information.

Finally, we knew nothing about java when we started writing this paper; we learned enough java to write the program mainly by studying Professor David Eck's fine online textbook [20].

\section{APPENDIX 6: CONSTRUCTION OF GLOW-IN-THE-DARK DIPPERCLOCKS}

In this appendix we describe our methods for making various kinds of glow-in-the-dark dipperclocks. One approach is to use an inkjet printer to print the dipperclock on glow-in-the-dark photo paper instead of ordinary paper. Reference [21] is an ad for a brand of glow-in-thedark photo paper that has worked well for us in making letter size (203 mm across) glowing dipperclocks. Lamination for durability and stiffness would be a good idea. Pocket size $(51 \mathrm{~mm}$ or $76 \mathrm{~mm})$ dipperclocks can also be made this way, but a brighter option for pocket size dipperclocks is to use glow paint rather than glow paper as follows:

1) Print a white-on-black dipperclock, then copy it onto a transparency appropriate for your copier. We have used a laser copier for this.

2) Paint the back of the dipperclock with glow-in-thedark paint. We have found that two or three coats typically works best. For the outer ring, we recommend the "ultra green V10" from [22]; according to the seller, this is the brightest glow paint available. For the inner ring, especially for the $51 \mathrm{~mm}$ dipperclock, we recommend "pure blue"; the ultra green V10 may be bright enough, when used in the inner ring, to blank out your central vision and make Polaris difficult to see. We recommend only water-based paint for safety reasons.

3) Cut away the transparency outside the dipperclock.

4) For durability and to keep little kids away from the glow paint, which can be hazardous if ingested, we recommend lamination. If you cannot see Polaris through the central hole, you can cut away the laminating material and transparency in the central hole.

\section{APPENDIX 7: FIELD TESTING}

We field tested our methods in and near Decatur, Illinois. Decatur is in the US Central time zone, with west longitude of $88.95^{\circ}$ according to [14], so according to Eq.1, the geographic correction for Decatur is

$$
\begin{aligned}
\mathrm{GC} & =4 \times(15 \times(-6)-(-88.95)) \\
& =4 \times(-1.05) \\
& =-4.2 \text { minutes }
\end{aligned}
$$

so we subtract about 4 minutes from our dipperclock time estimate.

Below, we summarize the results of our field tests

Test $1(6 / 17 / 10)$

Using a generic dipperclock, we estimated the time to be 9:30 p.m. CST, i.e. 10:30 p.m. CDT. Subtracting the 4 minute geographic correction (GC) gave 10:26 p.m. 
CDT. The actual time was about 10:22 p.m. CDT, so we were off by 4 minutes.

\section{Test 2 (5/29/11)}

Glow-in-the-dark dipperclocks, customized to Decatur, Illinois:

These tests were conducted with a full-size glow paper dipperclock (203 $\mathrm{mm}$ across) and a pocket-size transparency-glow paint dipperclock (51 $\mathrm{mm}$ across), with built-in geographic correction. We found that passing a small flashlight over each dipperclock for a few seconds charged them sufficiently for clear readings, and they remained sufficiently bright for the full hour duration of the test.

Full size glow paper dipperclock: This dipperclock was very easy to use, and gave consistently accurate readings, with errors ranging from a half hour to zero.

Pocket size painted transparency dipperclock: This was brighter than the glow paper dipperclock, but the numbers and letters were somewhat more difficult to read due to the small size. The accuracy was not quite as good as the accuracy with the full size glow paper dipperclock.

Test 3 (7/28/11)

Using a generic pocket-size Big Dipper dipperclock with a different tip star: At 10:11 p.m. CDT the pointer stars of the Big Dipper were behind trees, so we used the handle star next to the bowl, $\varepsilon$ UMa (aka Alioth), which has $\mathrm{RA}=12$ hours 54.1 minutes. This star gave a reading of about 7:30 p.m. CST, or 8:30 p.m. CDT. Here RA$11=1$ hour 54.1 minutes, so adding this to the reading as discussed at the end of Appendix 4 and doing the geographic correction we got about 8:30 + 1:54 - 0:04 $=$ 10:20 p.m. CDT, so we were off by 9 minutes.

\section{Test 4 (10/24/12)}

Finding the time, longitude, and latitude using a generic letter-size Big Dipper dipperclock: At about 8:30 p.m. CDT we used $\gamma$ Cas, the middle star of the $\mathrm{W}$ of Cassiopeia, which has right ascension 56.7 minutes, as the tip star. We got a reading of about 6:00 a.m. CST, i.e. about 7:00 a.m. CDT, on the outer ring of the dipperclock. Here RA $-11=-10$ hours 3.3 minutes. Adding this to our reading and doing the geographic correction we got about 7:00 a.m. - 10:3.3 - 0:04 = 8:53 p.m. CDT so we were off by about 23 minutes.

We also used Capella, aka $\alpha$ Aur, as the tip star and attempted to estimate our longitude as in Section 4. Capella has right ascension 5 hours 16.7 minutes, so RA $11=-5$ hours 43.3 minutes. Our time reading from the dipperclock was about 1:30 a.m., so converting this to DT, the time indicated by the pointer stars in the bowl of the Big Dipper, gives DT $=1: 30-5: 43.3=-4: 13$ (approximately). Universal Time is 5 hours ahead of CDT, so we had UT $=20: 30+5: 00=25: 30=1: 30$. From Eq.2 we get

$$
\begin{aligned}
\mathrm{L} & =(\mathrm{DT}-\mathrm{UT}) \times 15 \\
& =(-4: 13-1: 30) \times 15 \\
& =\left(-5 \frac{43}{60}\right) \times 15 \\
& =-85.75
\end{aligned}
$$

Thus the error in the longitude approximation is $85.75-(-88.95)=3.2$ degrees.

Finally, we estimated the latitude by the method in section 4 . When the dipperclock was held vertically with the hole beside the left eye and the $\mathrm{M}$ in the outer ring lined up with the northern point on the horizon, Polaris appeared to be close to $23 / 4$ in the outer ring. Multiplying by 15 gives 41.25 degrees as our estimate of the latitude. From [14] we have that the latitude of Decatur is 39.84 degrees, so we were off by about 1.4 degrees.

In general, our tests of various dipperclocks were satisfactory, with time errors usually less than 15 minutes.

\section{APPENDIX 8: FINDING THE TIME WITHOUT A DIPPERCLOCK (THE EQUINOX ESTIMATION METHOD)}

The equinox estimation method presented in this section involves computing an adjustment term based on the current date and adding this to a sidereal time estimate read from the Big Dipper.

First take the adjustment term to be 11 hours on September 22, the approximate date of the autumnal equinox. Subtract 2 hours for each month that has passed since September, and add/subtract 4 minutes for each day before/after the 22nd of the current month.

Look at Polaris and the Big Dipper, and imagine the outer ring of a dipperclock centered at Polaris and perpendicular to your line of sight to Polaris with the $\mathrm{M}$ at the top of the ring. Estimate the time on the imaginary outer ring at which the hour hand points, add the adjustment term, and add the geographic correction (if desired) to get your estimate of the local standard time.

As noted in [7], it is easy to get a little distortion in the time estimate, especially around 6 a.m. and 6 p.m. This occurs when the clockface is incorrectly imagined to be vertical, due to the fact that the hour hand lies in a plane which contains the axis of the earth, and a vertical clockface is not perpendicular to the axis of the earth. Imagining the clockface to be perpendicular to your line of sight to Polaris fixes this problem. The problem does not come up when using a dipperclock correctly since the dipperclock provides a properly tilted clockface.

\section{Example 3}

Suppose that on July 25 the stars are shown as in Figure 1 of section 1, with the clockface and hour hand seen only in our imagination. We compute the adjust- 
ment term as 11 hours $-10 \times 2$ hours $-3 \times 4$ minutes $=$ $-9: 12$. Adding this to the reading of $10: 30$ a.m. gives 1:18 a.m. local standard time, or 2:18 a.m. local daylight time. Actually, a careful measurement of Figure 1 gives a reading of about 10:45 a.m. instead of 10:30 a.m., giving a discrepancy of about 15 minutes in our results, but we do not have the luxury of doing such measurements in the field. This illustrates the effects of observational error; other types of error will be discussed in Appendices 11 and 12 .

We now describe two field tests using the equinox estimation method in Decatur, Illinois. As shown in Appendix 7, the geographic correction for Decatur is about -4 minutes.

Test 1 (6/17/10), equinox adjustment method

The adjustment for the equinox estimation method for 6/17/10 using the Big Dipper is

$$
\begin{aligned}
A & =11 \text { hours }-9 \times 2 \text { hours }+5 \times 4 \text { minutes } \\
& =-6 \text { hours } 40 \text { minutes }
\end{aligned}
$$

Given our reading of the imaginary clockface of 5:00 a.m. CDT, we had an estimated CDT of

$$
\begin{aligned}
\mathrm{CDT} & =5: 00+\mathrm{A}+\mathrm{GC} \\
& =5: 00-6: 40-0: 04 \\
& =-1: 44=22: 16,
\end{aligned}
$$

which was 10:16 p.m. CDT. The actual time was 10:22 p.m. CDT, so the error was 6 minutes.

Test $2(12 / 05 / 10)$, equinox adjustment method with $\gamma$ Cas

On $12 / 05 / 10$, we carried out an equinox estimation method exercise using $\gamma$ Cas as the tip of the hour hand. Using the right ascension of $\gamma$ Cas, namely about 57 minutes, in place of the 11 hours in the equinox estimation adjustment, we found the adjustment to be

$$
\begin{aligned}
A & =57 \text { minutes }-3 \times 2 \text { hours }+17 \times 4 \text { minutes } \\
& =-3 \text { hours } 55 \text { minutes }
\end{aligned}
$$

Given our reading of the imaginary clockface of 10:30 p.m. (=22:30 in 24-hour time), we had an estimated CST of

$$
\mathrm{CST}=22: 30-3: 55-0: 04=18: 31=6: 31 \text { p.m. }
$$

The actual time was 6:40 p.m. CST, so we were off by 9 minutes.

\section{APPENDIX 9: DISCUSSION OF THE MATHEMATICAL FOUNDATIONS REQUIRED FOR APPENDICES 10-12}

In this section, we assume we are located on the centerline of a time zone, so by Section 3, the geographic correction is 0 . The only clockface we will be using in this section will be the 24-hour counterclockwise clock- face with $\mathrm{M}$ at the top.

After introducing some basic notation, we derive the relationship between local standard time and time read from a star at the tip of the hour hand on the dipperclock, along with the number of days (including fractional days) since the last autumnal equinox (Eq.A5), which provides the foundation for the rest of our work.

a) We introduce the following notation:

$\mathrm{RA}=$ the right ascension of the star(s) we are using as the tip of the hour hand; for the pointer stars in the bowl of the Big Dipper, RA = 11 hours.

$\mathrm{LST}=$ the local standard time.

RAT $=$ the 24-hour time read from the star(s) at the tip of the hour hand when using a 24-hour counterclockwise clockface with $\mathrm{M}$ at the top.

b) Now we determine how long it takes the earth to complete one rotation on its axis. Imagine looking down on the solar system, with the earth revolving counterclockwise around the sun and rotating counterclockwise on its axis. Each 24 hours the earth makes one complete rotation, plus a little more, in order to keep the same face toward the sun at the same time each day. Over the course of one complete revolution around the sun, these little extra fractions of a rotation add up to one complete rotation. Since when viewed from below the earth is rotating toward the east (i.e. clockwise) it appears to us that the Big Dipper is rotating counterclockwise, and if we look at the Big Dipper again at the same time the next night, it will appear to have rotated a little counterclockwise from where it was the first night. To be precise, the earth makes one complete revolution around the sun in about 365.2422 24-hour days [5, p. 500], so the time for one revolution is $365.2422 \times 24$ hours. During this time the earth will make 365.2422 rotations on its axis plus one more. Thus the time for one rotation is $(365.2422 \times 24$ hours $) / 366.2422$, which is about 23 hours 56 minutes 4 seconds. Therefore, the solar day of 24 hours is about 3 minutes 56 seconds longer than the actual time it takes the earth to rotate once about its axis, relative to distant stars that we are not orbiting. This (approximately) 23 hours and 56 minute rotation period is called the sidereal day.

c) Now let's suppose that we look at the star(s) at the tip of the dipperclock's hour hand at the same LST on two consecutive nights. The second night, the tip stars will have returned to the same position after a sidereal day, but since we will be looking at them after a full solar day, they will be offset slightly from their position the previous night.

A little algebra shows that the time beyond one complete rotation of the earth until 24 hours is reached is $(1 / 366.2422) \times 24$ hours, and this is $1 / 365.2422$ of the time for one complete rotation.

Thus during our 24-hour period, the celestial hour 
hand will make one complete rotation, plus 1/365.2422 of a rotation. The complete rotation leaves RAT unchanged, and the $1 / 365.2422$ of a rotation increases RAT by $(1 / 365.2422) \times 24$ hours. Thus at the end of a 24-hour period, RAT will be greater by $(1 / 365.2422) \times$ 24 hours than it was at the beginning of the 24-hour period. LST will be the same at the end of the 24-hour period as at the beginning, so over our 24-hour period,

$$
\begin{aligned}
& \text { RAT }- \text { LST increases by } \\
& (1 / 365.2422) \times 24 \text { hours }(\text { per night }),
\end{aligned}
$$

which is about 3 minutes 56 seconds.

Now we need a starting point in order to map RAT into LST and complete our time conversion equation. We use the autumnal equinox (AE) as that starting point, at which

$$
\mathrm{LST}=\mathrm{RAT}+\mathrm{RA}
$$

because at the AE, 1) LST = the sidereal time [23] = the right ascension of whatever happens to be crossing the meridian at the time, and 2) the right ascension of whatever is crossing the meridian is always RAT + RA.

2) is true because it is true when the tip $\operatorname{star}(\mathrm{s})$ are directly above Polaris (they are crossing the meridian, and they have right ascension RA, but the 24-hour time read from the tip star(s) is RAT $=0$ ), and as time marches on from there both sides of the equation advance at the same rate, so the equation remains true.

From Eq.A3 it follows that over a period of d days, LST - RAT will decrease by $(24 / 365.2422) \times$ d hours. Combining this result with Eq.A4 gives the complete time conversion equation:

$$
\mathrm{LST}=\mathrm{RAT}+\mathrm{RA}-(24 \text { hours/365.2422 }) \times \mathrm{d}
$$

or

$$
\mathrm{LST}=\mathrm{RAT}+\mathrm{RA}-(3 \mathrm{~min} 56 \mathrm{sec}) \times \mathrm{d}(\text { approximately })
$$

where $d=$ the number of days (including fractional days) since the last autumnal equinox. Note that when $\mathrm{d}=$ 365.2422 , then we are (approximately) at the next autumnal equinox since the earth has returned to the same place in its orbit, the final term in Eq.A5 becomes 24 hours, and Eq.A5 becomes Eq.A4 again, as expected. Eq.A5 was also proved in a different form in [2] for the case of the Big Dipper, where RA $=11$.

\section{APPENDIX 10: DISCUSSION OF HOW THE VARIOUS NUMBERS AND HASH MARKS ARE PLACED AROUND THE DIPPERCLOCK}

To construct a dipperclock diagram you first draw three concentric circles to form two rings. You then need 24 hash marks on the outermost concentric circle, 15 degrees apart. The top hash mark is labeled (inside the outer ring) with the letter $\mathrm{M}$ standing for midnight, the next 11 hashmarks are labeled counterclockwise 1 through 11 for a.m. times, the next hashmark is labeled $\mathrm{N}$ for noon, and the remaining 11 hash marks are labeled counterclockwise 1 through 11 for p.m. times. Shorter hash marks are inserted between these to mark the half hours. In describing the location of these hash marks we will use the usual mathematical convention of measuring angles from the positive $\mathrm{x}$-axis, with counterclockwise angles being positive and clockwise angles being negative. Thus the location of the $\mathrm{M}$ hash mark is described by the angle 90 degrees.

We need 12 more hash marks on the inner and middle concentric circles, labeled clockwise with the first letters of the months in the inner ring. Placing these hash marks and letters correctly is much more involved than placing the hash marks and numbers in the outer ring. We will now derive the formulas for computing the position angles for these hash marks and letters for a dipperclock with given tip star(s), year-pair, time zone, and longitude (if a geographic correction is to be done).

From the given information we compute the following:

$\mathrm{RA}=$ the right ascension of the $\operatorname{star}(\mathrm{s})$ used as the tip of the hour hand; if we are using the pointer stars in the bowl of the Big Dipper, then RA $=11$ hours.

$\mathrm{AE}=$ an estimate of the autumnal equinox in local standard time for the first year of the year-pair, given in date (in September) + fractional day form.

LEAP $=1$ if the second year of the year-pair is a leap year, and LEAP $=0$ otherwise.

$\mathrm{LONG}=0$ if no geographic correction is to be done, and otherwise LONG = your longitude - the longitude of the centerline of your time zone, given in degrees + fractional degree form.

We now a) derive an equation which gives the position angle for the dates and their hash marks; b) show how to construct a dipperclock table; c) determine the dipperclock table for the generic dipperclock in Figure 2 of this paper; and d) justify the claim from the end of Appendix 4.

a) For now we will continue to assume we are located on the centerline of our time zone, so there is no geographic correction to be done. Referring to Eq.A5, we know that the date in the inner ring corresponding to $\mathrm{d}$ value $(\mathrm{RA} / 24) \times 365.2422$ must go at the bottom of the dipperclock when the $\mathrm{M}$ in the outer ring is at the top, because plugging this d value into Eq.A5 gives LST = RAT at that time. Thus at that time the correct clockface to use is the one with the $\mathrm{M}$ in the outer ring at the top, and with the given $\mathrm{d}$ value opposite the $\mathrm{M}$ as described; holding the dipperclock with this $d$ value at the bottom gives that clockface. 
Next we claim that to get from the position in the inner ring of the dipperclock corresponding to any $\mathrm{d}$ value to the position of $d+1$ we must move $(360 / 365.2422)$ degrees clockwise in the inner ring. To see this, suppose we look at the tip star(s) at the date and time corresponding to $\mathrm{d}$, so we will be holding the dipperclock with $d$ at the bottom. If we look at the tip stars again 24 hours later, so that $d+1$ will be at the bottom, then by earlier work in part c) of Appendix 9, the celestial hour hand will be advanced by $1 / 365.2422$ of a rotation compared to where it was at the beginning of the 24-hour period. $1 / 365.2422$ of a 360 -degree rotation is (360/365.2422) degrees. LST will be the same at the end of the 24-hour period as at the beginning of the period, so we will need to have rotated the dipperclock counterclockwise (360/365.2422) degrees to keep the celestial hour hand pointing at the same time in the outer ring, so the $d+1$ position will be $(360 / 365.2422)$ degrees clockwise from the $\mathrm{d}$ position, as claimed. By proportionality, getting from position $\mathrm{d} 1$ to position $\mathrm{d} 2$ (with $0 \leq \mathrm{d} 1 \leq \mathrm{d} 2 \leq 365.2422)$ requires moving $(\mathrm{d} 2-\mathrm{d} 1) \times$ (360/365.2422) degrees clockwise on the dipperclock.

By the definition of $d$, when $d=0$, we are at the last autumnal equinox, and by the first claim in subsection (a), when $d=(\mathrm{RA} / 24) \times 365.2422$ we are at the bottom of the dipperclock (with the $\mathrm{M}$ in the outer ring at the top), so the distance moved clockwise around the dipperclock from $\mathrm{d}=0$ to $\mathrm{d}=(\mathrm{RA} / 24) \times 365.2422$ is

$$
((\mathrm{RA} / 24) 365.2422-0) \times(360 / 365.2422)
$$

degrees, which equals $(360 / 24)$ RA degrees $=15 \mathrm{RA}$ degrees. Thus we have the remarkable fact that whatever date and time we are using for the last autumnal equinox, it corresponds to a point on the dipperclock that is 15RA degrees counterclockwise from the bottom of the dipperclock. If we move from the positive $\mathrm{x}$-axis to the autumnal equinox position on the dipperclock instead of from the bottom of the dipperclock we are moving $90^{\circ}$ less, so the autumnal equinox has position angle 15RA $90^{\circ}$.

Now consider the location (i.e. position angle) $\theta$ of midnight of the first day of a month following the value we are using for the autumnal equinox. Let $d$ be the number of days plus fractional day that this time is past the autumnal equinox. Then by the work at the end of the second paragraph above Eq.A6 with $\mathrm{d} 1=$ the autumnal equinox, $\mathrm{d} 2$ = midnight of the first day of the month, so $d=d 2-d 1$, getting to location $\mathrm{d} 2$ from the autumnal equinox on the dipperclock requires a clockwise move of $d \times(360 / 365.2422)$ degrees. Clockwise is the negative direction, so we are changing the position angle by $-\mathrm{d} \times(360 / 365.2422)$ degrees, so

$$
\theta=15 \mathrm{RA}-90^{\circ}-(360 / 365.2422) \times \mathrm{d} \text { degrees (A7) }
$$

In deriving Eq.A7 we made the assumption that no geographic correction was done, so $\mathrm{LONG}=0$. Now suppose a geographic correction is to be built into the dipperclock, where LONG $=\mathrm{L}-15 \times(\mathrm{LST}-\mathrm{UT})$ (using the notation of sections 3 and 4). The final result is then

$$
\theta=15 \mathrm{RA}-90^{\circ}-(360 / 365.2422) \times \mathrm{d}-\text { LONG degrees }
$$

Let's justify this for the case where you are one degree of longitude east of the centerline of your time zone. (The argument for other cases is similar.) Then $\mathrm{LONG}=$ 1. Statement (A1a) in Appendix 2 tells us to subtract 4 minutes from your time estimate, but this can be accomplished by rotating the dipperclock one degree counterclockwise. To compensate for this rotation, the dates and hash marks in the inner ring need to be rotated one degree clockwise, so the same date will be at the bottom. This can be done by subtracting one degree from each of the locations in Eq.A7 and Eq.A8 does precisely that.

b) Now we are ready to construct a dipperclock table. The table is a $12 \times 3$ matrix.

The first column of the table contains the numeric indicators of the months in the order 10.0, 11.0, 12.0, 1.0, 2.0, $\cdots, 9.0$.

Each entry in the second column contains the number $\mathrm{d}$ of days plus fractional day (at midnight) that the first day of the month in column 1 is past the value being used for the autumnal equinox.

Each entry in the third column contains the position angle $\theta$ given by Eq.A8 using the d value to the left.

To compute the first element in the second column, first convert October 1 at midnight to (imaginary) September 31 at midnight, or September 32.0, so we get 32.0 - AE. Since there are 31 days from October 1 to November 1, the second element in the second column $=$ the first element in the second column +31.0 . We continue down the second column this way, except the sixth element in the second column $=$ the fifth element in the second column $+28+$ LEAP to allow for a possible leap day in the second year of the year-pair. Finally, the elements in the third column are computed from Eq.A8 with the $\mathrm{d}$ values taken from the second column.

c) For the generic dipperclock shown in Figure 2 of this paper, we used $\mathrm{RA}=11, \mathrm{AE}=$ September 22 at midnight LST, LEAP $=0, \mathrm{LONG}=0$. Table $\mathbf{1}$ below is the table corresponding to these choices that provided the position angles for Figure 2.

d) In Appendix 4 we claimed that it is possible to use a dipperclock constructed for the Big Dipper with a new tip star with right ascension RA; just take a reading with the new tip star and add RA -11 hours. To see this, suppose RA $>11$; the case $\mathrm{RA}<11$ is similar. Consider a new dipperclock constructed for the new tip star. Re- 
Table 1. Inner ring locations for the generic dipperclock (Figure 2).

\begin{tabular}{ccc}
\hline month & d & $\boldsymbol{\theta}$ \\
\hline 10.0 & 9.0 & 66.1 \\
11.0 & 40.0 & 35.6 \\
12.0 & 70.0 & 6.0 \\
1.0 & 101.0 & -24.6 \\
2.0 & 132.0 & -55.1 \\
3.0 & 160.0 & -82.7 \\
4.0 & 191.0 & -113.3 \\
5.0 & 221.0 & -142.8 \\
6.0 & 252.0 & -173.4 \\
7.0 & 282.0 & -203 \\
8.0 & 313.0 & -233.5 \\
9.0 & 344.0 & -264.1 \\
\hline
\end{tabular}

placing RA by 11 in Eq.A8 and subtracting from the original Eq.A8, the updated position angles $\left(\theta^{\prime}\right)$ for the new dipperclock are

$$
\theta^{\prime}=\theta+15 \times(\mathrm{RA}-11) \text { degrees, }
$$

where $\theta$ represents the position angles found for a dipperclock based on the Big Dipper. Since the position angle for the $\mathrm{d}$ value formerly at the bottom of the dipperclock has increased by $15 \times(\mathrm{RA}-11)$ degrees, returning this $d$ value to the bottom of the new dipperclock rotates the new dipperclock $15 \times(\mathrm{RA}-11)$ degrees clockwise with respect to the actual Big Dipper dipperclock. This is a clockwise rotation of $15 \times(\mathrm{RA}-11) / 360$ of a complete circle, or $(\mathrm{RA}-11) / 24$ of a complete circle, or RA - 11 hours on the outer ring. The new tip star is pointing at the correct time on the new dipperclock, but due to the rotation this correct time is RA - 11 hours counterclockwise from where the new tip star is pointing on the actual Big Dipper dipperclock. Thus the time pointed at by the new tip star on the actual Big Dipper dipperclock is RA - 11 hours too early, so we need to add RA - 11 hours to get the correct time.

\section{APPENDIX 11: ACCURACY OF THE DIPPERCLOCK}

In this section we assume that the correct geographic correction from section 3 has been done. We consider the following possible sources of error: a) error in Eq.A5; b) error due to using an inaccurate value for the autumnal equinox; c) error in using a dipperclock constructed with the second year being a non-leap year to estimate times with the second year being a leap year (or vice versa); d) error due to necessarily thinking of the dates in the inner ring as being discrete; and e) observational error.

a) Eq.A5 is quite accurate in terms of its theoretical error (by which we mean the error in the equation itself, irrespective of observational errors). This is because the only major sources of error in Eq.A5 are rounding and to a much lesser extent, effects like the precession cycle of earth's rotation axis, which has a period of approximately 26,000 years. See [5] for further discussions of this and other small sources of error. Consequently, we can say very conservatively that Eq.A5 is accurate to less than one minute.

b) We note from Eq.A6 that an error of one day in d results in a less than 4 minute discrepancy in our estimate of LST. For the generic dipperclock, we use a default value of midnight on September 22 (LST) for the autumnal equinox (AE), as a characteristic average. For instance, according to [15], for the years 2000 to 2020 the maximum that an autumnal equinox falls short of midnight September 22 is 10 hours 29 minutes (for 2020) and the maximum in those years that an autumnal equinox goes beyond midnight September 22 is 10 hours 47 minutes (for 2003). Thus, in any year-pair with first year between 2000 and 2020, the discrepancy between the autumnal equinox (UT) and midnight September 22 (UT) is less than 12 hours. There is also a maximum 12 hour discrepancy in converting the UT autumnal equinox we have been discussing to the autumnal equinox for your time zone, which is what appears in Eq.A5, giving a discrepancy of your autumnal equinox from midnight September 22 of less than 24 hours, which equals one day. Multiplying this by 4 minutes, we can conservatively say that using midnight September 22 LST as our value for the autumnal equinox should cause an error in our estimate of the current local standard time of less than 4 minutes.

c) Whether or not the second year is a leap year will make a difference of 1 in $\mathrm{d}$ for the months March $\cdots$, September, and this difference can also affect our estimated time by less than 4 minutes for these months. Recall that our generic dipperclock was constructed under the assumption that the second year is not a leap year.

d) Think of a dipperclock in use. As time progresses, the hour hand of the clock rotates continuously counterclockwise. The clockface rotates also, but not continuously, due to changes in the date at the bottom of the inner ring. For example, from 12:01 a.m. August 7 to 11:59 p.m. August 7, the clockface does not rotate, but from 11:59 p.m. August 7 to 12:01 a.m. August 8, the clockface rotates because the date at the bottom of the inner ring changes by one day. This corresponds to a less than one day change in the $d$ value at the bottom of the dipperclock when compared to the continuous and uniform rotation situation, which causes a change of less than 4 minutes in the time read from the dipperclock. 
This source of error would not be present if the dipperclock rotated continuously and uniformly, but for that to happen the point at the bottom of the inner ring would have to involve the time of day as well as the month and day, and we do not know the time of day, since that is what we are trying to find by using the dipperclock! Also, even if we knew the time of day, very few humans could place the date at the bottom of the dipperclock accurately enough for the time of day to make a difference.

Thus, the total theoretical error for the generic dipperclock will be less than $1+4+4+4$ minutes, or 13 minutes, at least when the first year of the year-pair is between 2000 and 2020. For a custom dipperclock there is no error for the autumnal equinox or leap years, so the theoretical error will be less than $1+4$ minutes, or 5 minutes.

Remark: Comparison of theoretical maximum error to actual error for the 2010-2011 year-pair: From [15], the autumnal equinox for 2010 occurs on September 23 at 3:09 in UT, which is September 22 at 21:09 (or 9:09 p.m.) CST in the US Central time zone. The difference between this AE value and the default value of September 22 at midnight used for the generic dipperclock is 2 hours 51 minutes $=0.12$ days, which corresponds to a discrepancy of less than $0.48 \mathrm{~min}=29 \mathrm{sec}$ in Eq.A5. Since the 4-minute leap year error does not come into play for this year-pair, the actual theoretical error estimate for our generic dipperclock in 2010-2011 is only about $51 / 2$ minutes instead of 13 minutes. This explicitly illustrates that our theoretical maximum tends to be very conservative.

e) There are four main kinds of observational error one can introduce when using a dipperclock: 1) not holding the dipperclock with the current date exactly on the bottom; 2) not having Polaris at the center; 3) not holding the dipperclock perpendicular to the line of sight direction to Polaris; and 4) not reading exactly where the hour hand is pointing. From our testing, observational error seems to be the largest source of error in using a dipperclock.

\section{APPENDIX 12: ACCURACY OF THE EQUINOX ESTIMATION METHOD}

In this section we assume that the proper geographic correction from Section 3 has been done.

Here we analyze the equinox estimation method when we are using the pointer stars in the bowl of the Big Dipper as the tip of the hour hand (so RA $=11$ ), and we are using our default autumnal equinox value of midnight September 22 LST.

The equinox estimation method has the possibility of errors in Eq.A5 itself (less than 1 minute) and errors due to an inaccurate choice for the autumnal equinox (less than 4 minutes for year-pairs with first year in the range
2000-2020) as we saw in Appendix 11.

However, there is an additional source of potential theoretical error; namely, the equinox estimation method of approximating the terms $11-(24$ hours/365.2422 $) \times$ d in Eq.A5 by using our adjustment procedure. We will now estimate this error. To do this we first suppose the second year is not a leap year and compute Table 2 below with the following five columns:

The first column contains the dates 9/22, 9/30, 10/1, $10 / 31,11 / 1 \ldots 8 / 31,9 / 1$, and $9 / 22$, all at midnight. The second column contains the value of $d$, which is the number of days (including fractional days) since September 22 at midnight. The third column contains the time adjustment for the equinox estimation method. The fourth column contains the correct adjustment from Eq.A5, namely $11-(24$ hours/365.2422 $) \times d$, and the fifth column is obtained by subtracting the fourth column from the third column.

In Table 2, all times are rounded to the nearest minute. The largest absolute value of elements shown in column 5 is 7 minutes. There can be no larger absolute value of

Table 2. Maximum theoretical error due to equinox estimation computation.

\begin{tabular}{|c|c|c|c|c|}
\hline $9 / 22$ & 0 & 11 & 11 & 0 \\
\hline $9 / 30$ & 8 & $10: 28$ & $10: 28$ & 0 \\
\hline $10 / 1$ & 9 & $10: 24$ & $10: 25$ & $-: 01$ \\
\hline $10 / 31$ & 39 & $8: 24$ & $8: 26$ & $-: 02$ \\
\hline $11 / 1$ & 40 & $8: 24$ & $8: 22$ & :02 \\
\hline $11 / 30$ & 69 & $6: 28$ & $6: 28$ & 0 \\
\hline $12 / 1$ & 70 & $6: 24$ & $6: 24$ & 0 \\
\hline $12 / 31$ & 100 & $4: 24$ & $4: 26$ & $-: 02$ \\
\hline $1 / 1$ & 101 & $4: 24$ & $4: 22$ & :02 \\
\hline $1 / 31$ & 131 & $2: 24$ & $2: 24$ & 0 \\
\hline $2 / 1$ & 132 & $2: 24$ & $2: 20$ & $: 04$ \\
\hline $2 / 28$ & 159 & $: 36$ & $: 33$ & :03 \\
\hline $3 / 1$ & 160 & $: 24$ & $: 29$ & $-: 05$ \\
\hline $3 / 31$ & 190 & $-1: 36$ & $-1: 29$ & $-: 07$ \\
\hline $4 / 1$ & 191 & $-1: 36$ & $-1: 33$ & $-: 03$ \\
\hline $4 / 30$ & 220 & $-3: 32$ & $-3: 27$ & $-: 05$ \\
\hline $5 / 1$ & 221 & $-3: 36$ & $-3: 31$ & $-: 05$ \\
\hline $5 / 31$ & 251 & $-5: 36$ & $-5: 30$ & $-: 06$ \\
\hline $6 / 1$ & 252 & $-5: 36$ & $-5: 34$ & $-: 02$ \\
\hline $6 / 30$ & 281 & $-7: 32$ & $-7: 28$ & $-: 04$ \\
\hline $7 / 1$ & 282 & $-7: 36$ & $-7: 32$ & $-: 04$ \\
\hline $7 / 31$ & 312 & $-9: 36$ & $-9: 30$ & $-: 06$ \\
\hline $8 / 1$ & 313 & $-9: 36$ & $-9: 34$ & $-: 02$ \\
\hline $8 / 31$ & 343 & $-11: 36$ & $-11: 32$ & $-: 04$ \\
\hline $9 / 1$ & 344 & $-11: 36$ & $-11: 36$ & 0 \\
\hline $9 / 22$ & 365 & - & $-12: 59$ & $-: 01$ \\
\hline
\end{tabular}


an element in column 5 for any integer $d$ since as we move from $\mathrm{d}$ to $\mathrm{d}+1$, staying within one month, column 3 decreases by 4 minutes while column 4 decreases by a lesser amount (namely by 24/365.2422 hours, or about 3.94 minutes), so even with rounding, column 5 is non-increasing for integer values of $\mathrm{d}$ within any one month. Thus, the largest absolute value of any number in column 5 for integer d will occur on the first or last day of that month, and so will already be in Table 2 . We note that the average absolute value of the numbers in column 5 is $0.70 / 26$, or about 0.03 , which is less than half of the maximum absolute value.

For non-integer $d$ values, there is another possible effect. For example, suppose we put in another line in Table 2 for 1:00 a.m. on March 31. Column 3 will still be at $-1: 36$ since we will be in March 31 , but we will have $\mathrm{d}=189+1 / 24$, and computing column 4 gives $-1: 25$. Thus, the value in column 5 is -:11, which is the worst case scenario.

Although Table 2 was constructed for the second year being a non-leap year, one can also construct another table for the second year being a leap year. In that table, the error behavior is actually better. Thus, we conclude that the maximum theoretical error in our time estimate via the equinox estimation method is 11 minutes. Putting this together with our previous estimates, we find that the theoretical error in the equinox estimation method under the assumptions given in the second paragraph of this section, with first year of the year-pair between 2000 and 2020 , is less than 16 minutes $(1+4+11)$.

This is a conservative estimate. For one thing, our equinox estimation method implicitly uses September 22 at midnight as its value for the autumnal equinox, just as our generic dipperclock does, so the Remark near the end of Appendix 11 applies and indicates that the error due to an inaccurate choice of the value for the autumnal equinox is typically much less than 4 minutes. The 11 minute estimate in the previous paragraph is also unlikely to be achieved, so the 16 minute maximum theoretical error estimate is far larger than what you are likely to find in practice.

Using this method, the observational error will be even more extreme than for a dipperclock because we will be employing an imagined clock face instead of a dipperclock for our time estimate, and this is a considerable disadvantage. The field tests given in appendices 7 and 8 offer some insights into the characteristic total errors we encountered when using both the equinox estimation method as well as generic and customized dipperclocks. 